\title{
A Multichannel Spatial Compressed Sensing Approach for Direction of Arrival Estimation
}

\author{
Aris Gretsistas and Mark D. Plumbley \\ Queen Mary University of London \\ Centre for Digital Music \\ Mile End Road, E1 4NS, London, UK \\ aris.gretistas@elec.qmul.ac.uk
}

\begin{abstract}
In this work, we present a direction-of-arrival (DOA) estimation method for narrowband sources impinging from the far-field on a uniform linear array (ULA) of sensors, based on the multichannel compressed sensing (CS) framework. We discretize the angular space uniformly into a grid of possible locations, which is much larger than the number of sensors, and assume that only a few of them will correspond to the active sources. As long as the DOAs of the sources are located at a few locations on the angular grid, they will share a common spatial support. To exploit this joint sparsity, we take several time snapshots and formulate a multichannel spatial compressed sensing (SM-CS) problem. Simultaneous Orthogonal Matching Pursuit (SOMP) is used for the reconstruction and the estimation of the angular power spectrum. The performance of the proposed method is compared against standard spectral-based approaches and other sparsity based methods.
\end{abstract}

Keywords: array signal processing, direction of arrival estimation, sparse representations, compressed sensing, joint sparsity.

\section{Introduction}

One of the main objectives in array processing is to estimate the spatial energy spectrum and therefore determine the number and location of the sources of energy. This source localization problem using sensor arrays, usually referred to as direction-of-arrival (DOA) estimation, arises in many applications such as radar, communications, seismology and sonar.

Among the various methods that has been proposed for the DOA estimation problem, conventional (or Bartlett) beamforming provides a spatial spectral estimate, which suffers from its inability to resolve closed spaced sources [1]. These resolution limitations of conventional beamforming can be overcome using Capon's method (MVDR) or the MUSIC algorithm. Both techniques can achieve superresolution by focusing on a small number of "search directions"

\footnotetext{
* This research is supported by ESPRC Leadership Fellowship EP/G007144/1 and Platform Grant EP/045235/1, and EU FET-Open Project FP7-ICT-225913 "SMALL".
} 
where the sources are present. Therefore, this implies some underlying sparsity in the spatial domain.

The emerging field of sparse representations has given renewed interest to the problem of source localization. The concept of spatial sparsity for DOA estimation was first introduced in [2], where it was shown that the source localization problem can be cast as a sparse representations recovery problem in a redundant dictionary using the $\ell_{1}$-SVD method. More recently, in a similar manner, spatial sparsity was linked to the theoretical results of the compressed sensing (CS) framework, utilizing a spatial CS approach for DOA estimation [3]. That work demonstrated that a simple implementation of the CS-based approach can achieve high angular resolution.

In this paper, we attempt to further exploit the CS framework by formulating the problem of DOA estimation of far-field narrowband sources as a multichannel spatial compressed sensing (MS-CS) or distributed compressed sensing reconstruction problem using the joint sparsity model of common sparse support [4]. To achieve this, we uniformly discretize the space into a grid of possible angles of arrival, assuming that only a few of them will correspond to real source locations. A uniform linear array is used to capture the observed time snapshots of the impinging far-field sources.

Assuming that the DOAs of the sources will share a common spatial support at some locations on the grid, we observe several time snapshots and attempt to solve a mixed norm minimization problem. The simultaneous Orthogonal Matching Pursuit (SOMP) algorithm [5] is used for the reconstruction of the common sparse support and yields an estimate of the angular signal energy spectrum, in which the high energy spikes correspond to the sources angles of arrival.

We will show that the proposed method can achieve superresolution and under certain conditions outperform the single-channel spatial CS (SS-CS) approach, while being less vulnerable to highly correlated sources or limited data when compared to MUSIC algorithm.

\section{Distributed Compressed Sensing with common sparse support}

Consider $L$ linear measurement sensors that each of them obtains $M$ samples or observations of the discrete-time signal of interest $\mathbf{x}=\left[x_{1}, \ldots, x_{N}\right]^{T}$, where $M<N$. The observations or measurements vector can be written as

$$
\mathbf{Y}=\mathbf{\Phi} \mathbf{X}
$$

where $\boldsymbol{\Phi}$ is a $M \times N$ matrix representing the measurement process, $\mathbf{Y}$ is the $M \times L$ measurements and $\mathbf{X}$ an and $N \times L$ matrix.

The main assumption is that the discrete-time signal $\mathbf{x}$ is sparse in some domain $\mathbb{R}^{N}$ and can be decomposed and accurately represented in the square basis $\Psi \in \mathbb{R}^{N \times N}$. The resulting system of linear equations

$$
\mathbf{Y}=\mathbf{\Phi X}=\boldsymbol{\Phi} \Psi \mathbf{S}=\mathrm{AS}
$$


is underdetermined as the dimensionality of the sparse $N \times L$ matrix $\mathbf{S}$ is larger than the dimensionality of the observed signal space. In the case that $L=1$, the problem is reduced to single channel standard compressed sensing problem.

However, considering the common sparse support distributed compressed sensing model introduced in [4], sparsity is enforced across the columns of the sparse matrix $\mathbf{S}$. Therefore, we need to solve the mixed $\ell_{1,2}$ norm minimization problem defined as:

$$
\min \|\mathbf{S}\|_{1,2}=\sum_{j=0}^{N}\left\|\mathbf{S}^{j}\right\|_{2} \quad \text { such that } \quad \mathbf{Y}=\mathbf{A} \mathbf{S}
$$

The above optimization problem is convex and can be solved using interiorpoint methods. Alternatively, greedy approaches as simultaneous Orthogonal Matching Pursuit (SOMP) [5], which is the joint sparsity version of Orthogonal Matching Pursuit (OMP) [6] can provide faster convergence for an approximate solution.

The theoretical recovery results of compressed sensing have been generalized in the multiple sensor case [7]. Therefore, if matrix $\mathbf{A}$ obeys the restricted isometry property (RIP)

$$
\left(1-\delta_{k}\right)\|\mathbf{s}\|_{2}^{2} \leq\|\mathbf{A} \mathbf{s}\|_{2}^{2} \leq\left(1+\delta_{k}\right)\|\mathbf{s}\|_{2}^{2}
$$

where $\delta_{k}$ is a small constant and if $\delta_{2 k}<\sqrt{2}-1$ then the the solution $\overline{\mathbf{S}}$ obeys

$$
\|\mathbf{S}-\overline{\mathbf{S}}\|_{F} \leq C_{0} k^{-1 / 2}\left\|\mathbf{S}-\hat{\mathbf{S}}^{(k)}\right\|_{1,2}
$$

for some constant $C_{0}$, where $\mathbf{S}^{(k)}$ is the matrix $\mathbf{S}$ with all but the largest $k$ components set to 0 and $\|\cdot\|_{F}$ is the frobenius matrix norm. The recovery will be exact as long as $|\operatorname{supp} \mathbf{X}| \leq k$.

Put another way, if the measurement matrix $\mathbf{\Phi}$ and the representation ma$\operatorname{trix} \boldsymbol{\Psi}$ are sufficiently incoherent and the number of measurements $M<N$ is sufficiently large, the original signal can be recovered with high probability. It is well known that random Gaussian or Bernoulli matrices obey the RIP condition with overwhelming probability as long as the number of measurements for each sensor is $M \geq C k \log (N / k)$ [8]. In the case of partial Fourier matrices the bound on measurements will be $M \geq C k \log ^{4}(N)$ [9].

However, one can notice that these bounds are the same for the single channel compressed sensing problem. The above results based on RIP follow the worst case analysis scenario and therefore predict no performance gain resulting in theoretical equivalence between single and multi-channel sparsity. In practice, the worst case scenario is not always the case and empirical results have shown that as the number of channel $L \rightarrow \infty, k+1$ measurements per channel will be sufficient for the exact reconstruction. As stated in [10], average case analysis can give more insight into joint sparsity and its superiority to the standard single channel case. Average case analysis provides the theoretical framework for joint sparsity and proves that the probability of recovery failure gets increasingly smaller as the number of channels increases [7]. 


\section{Source localization via Multichannel Compressed Sensing}

Consider $k$ narrowband sources with known central frequency $f$ propagating plane waves from the far-field which impinge on an uniform linear array (ULA) of $M$ sensors with inter-sensor spacing $d$. The sources arrive on the array from the unknown angles $\theta_{1}, \theta_{2}, \ldots, \theta_{k}$ that we wish to estimate. The linear array response to the impinging plane waves can be expressed as :

$$
\boldsymbol{a}\left(\theta_{n}\right)=\left[1, e^{-j \omega_{c} \tau_{2}\left(\theta_{n}\right)}, \ldots, e^{-j \omega \tau_{M}\left(\theta_{n}\right)}\right]
$$

or after the substitution of $\omega=2 \pi f$ and $\tau_{p}=p d \cos \left(\theta_{n}\right) / c$, where $d$ is the sensor spacing which is chosen at half the wavelength $d=\lambda / 2, \lambda=c / f$ is the wavelength and $c$ is the speed of the propagation, we end up with the formula:

$$
\boldsymbol{a}\left(\theta_{n}\right)=\left[1, e^{-j \pi \cos \left(\theta_{n}\right)}, \ldots, e^{-j \pi \cos \left(\theta_{n}\right)(M-1)}\right]
$$

Therefore, the array output can be expressed as:

$$
\mathbf{y}(t)=\mathbf{A}(\theta) \mathbf{s}(t)+\mathbf{n}(t)
$$

whre $\boldsymbol{s}(t)$ is a $k \times 1$ vector containing the $k$ sources, $\boldsymbol{y}(t)$ is a $M \times 1$ vector of the measurements of the array sensors, $\boldsymbol{n}(t)$ is the noise vector and $\mathbf{A}(\theta)$ is a $M \times k$ matrix containing the array responses for the $k$ plane waves

$$
\mathbf{A}(\theta)=\left[\boldsymbol{a}\left(\theta_{1}\right), \ldots, \boldsymbol{a}\left(\theta_{k}\right)\right] .
$$

The above problem, presented in equation (8), is an overdetermined or evendetermined system of linear equations as long as $M \geq k$ and can be easily solved by inverting the matrix when the DOAs of the sources are known. However, the DOAs are unknown and need to be estimated. Following a spatial sparsity approach as in [2], we can uniformly discretize the bearing space into $N>>k$ possible angles of arrival and construct a redundant matrix of $N$ atoms corresponding to the array responses of the respective angles of arrival

$$
\boldsymbol{\Phi}=\left[\boldsymbol{a}\left(\theta_{1}\right), \ldots, \boldsymbol{a}\left(\theta_{N}\right)\right] .
$$

This discretization provides the sparsity requirement of the compressed sensing framework. However as has already been stated above, in order for the reconstruction to be exact the chosen sensing matrix must obey the RIP condition and contain elements that are not correlated. According to the recent spatial compressed sensing approach, the source localization problem can be formulated as:

$$
\mathbf{y}(t)=\mathbf{\Phi} \mathbf{s}(t)+\mathbf{n}(t)
$$

where $\boldsymbol{\Phi}$ can be viewed as the sensing system of the compressed sensing problem. Considering that the expansion matrix $\boldsymbol{\Psi}$ is the Dirac matrix, the resulting pair of matrices is highly incoherent [3]. In fact, it has been proven that the pair of 
Dirac basis and Fourier basis exhibits maximal incoherence [8] and therefore the compressed sensing framework can be applied to the above problem for the one time sample case and can be solved by Basis Pursuit (BP) $\ell_{1}$ minimization [11].

However, for the multiple time samples case we intend to follow a MS-CS approach under the common sparse support model rather than solving the BP problem for each time sample separately. In that case the problem can be formulated:

$$
\mathbf{Y}=\mathbf{\Phi} \mathbf{S}+\mathbf{N} .
$$

We are interested in enforcing spatial sparsity and not temporal, so we need to solve the mixed norm minimization problem for noisy data:

$$
\min \|\mathbf{S}\|_{1,2} \text { such that }\|\mathbf{\Phi S}-\mathbf{Y}\|_{F} \leq \epsilon .
$$

The mixed $\ell_{1,2}$ norm minimization can be solved using linear programming methods e.g. interior-point methods. Our approach will focus on the fast convergence of joint sparsity greedy approaches and more specifically the SOMP algorithm will be used. The proposed method assumes that the additive noise at the sensors of the array is white gaussian noise and the standard deviation $\sigma$ is known. Therefore, the stopping criteria for the SOMP algorithm can be adjusted according to the noise level, very much like in [12].

It has already been stated that the compressed sensing worst case analysis shows theoretical equivalence for the single and multi-channel case. However, the worst case can occur when there is no additional information on the support from the multiple channels. This implies that each of them will contain the same information, which typically is not the case in the source localization problem, as the received signals' time samples are expected to vary. Consequently, the proposed multi-channel spatial sparsity model exploits the joint support and is expected to require fewer sensors than the $M \geq C K \log ^{4}(N)$ required for the standard compressed sensing approach.

\section{Experimental Results}

In this section we present experimental results of the proposed method of joint spatial sparsity compressed sensing. The performance of our method is compared against the array processing spectral based approaches of conventional beamforming and MUSIC and the spatial sparsity compressed sensing method. We consider five narrowband far-field sources located at angles $30^{\circ}, 45^{\circ}, 50^{\circ}$, $100^{\circ}$ and $130^{\circ}$ respectively. The plane waves impinge on an ULA of $M=40$ sensors equally spaced at the half wavelength $d=\lambda / 2$. The sensors acquire $L=200$ time snapshots of the superimposed signals and the signal-to-noise ratio (SNR) is $10 \mathrm{~dB}$. The additive noise is gaussian and MS-CS method assumes the knowledge of its standard deviation for the adjustment of the stopping criteria of SOMP. Empirically, we have found that a good choice is $0.8 \sigma$. The angular resolution is $1^{\circ}$ and subsequently considering omnidirectional sensors the number of potential locations is $N=180$. 
Figure 1 illustrates the estimated angular power spectrum of all tested methods. Conventional beamforming exhibits the worst performance in terms of resolution. MUSIC and both SS-CS and MS-CS methods are able to estimate the DOAs. However, the proposed method gives the sharper peaks and showcases the best performance.

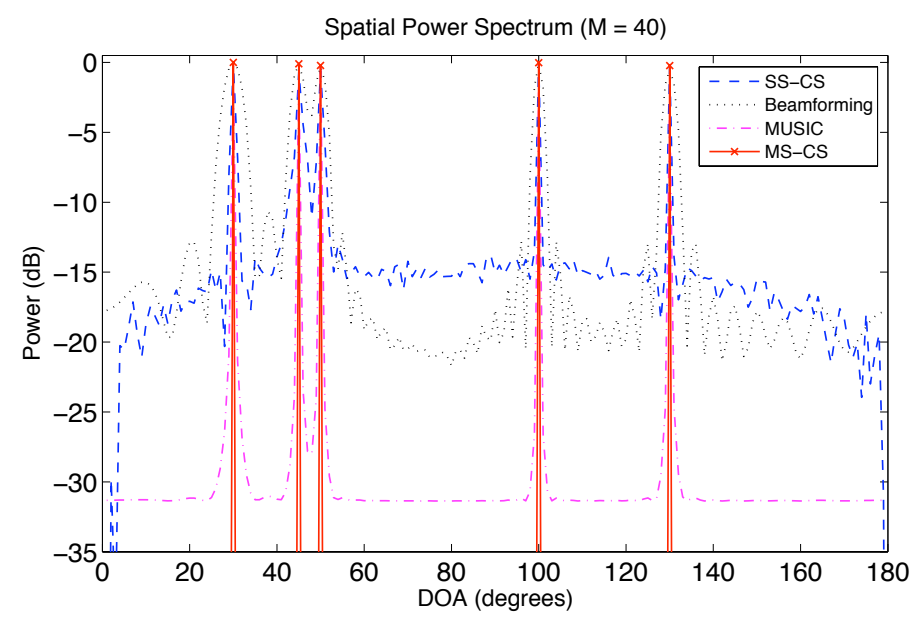

Fig. 1. Comparison of the proposed method (MS-CS) with the single channel CS (SSCS) approach, conventional beamforming and MUSIC algorithm. The number of sensors is $M=40$ and the SNR is $10 \mathrm{~dB}$.

Next we reduce the number of sensors to $M=30$ and repeat the same experiment. Figure 2 shows that for the two closed spaced sources the performance of the SS-CS method decreases significantly. However, the performance of MS-CS method and MUSIC algorithm remain intact.

The elapsed time of each method is shown in Table 1. Clearly, the spectral based methods are faster than the sparsity based approaches. However, the proposed method improves on the convergence speed of the single channel case by exploiting the joint spatial sparsity.

Table 1. Elapsed time for the spatial spectrum estimation

\begin{tabular}{|c|c|c|}
\hline No of sensors & Beamforme & MUSIC SS-CS MS-CS \\
\hline 30 & $0.0041 \mathrm{~s}$ & $0.0054 \mathrm{~s} 1.1040 \mathrm{~s} 0.0401 \mathrm{~s}$ \\
\hline 40 & $0.0047 \mathrm{~s}$ & $0.0068 \mathrm{~s} 1.5831 \mathrm{~s} 0.0705 \mathrm{~s}$ \\
\hline
\end{tabular}



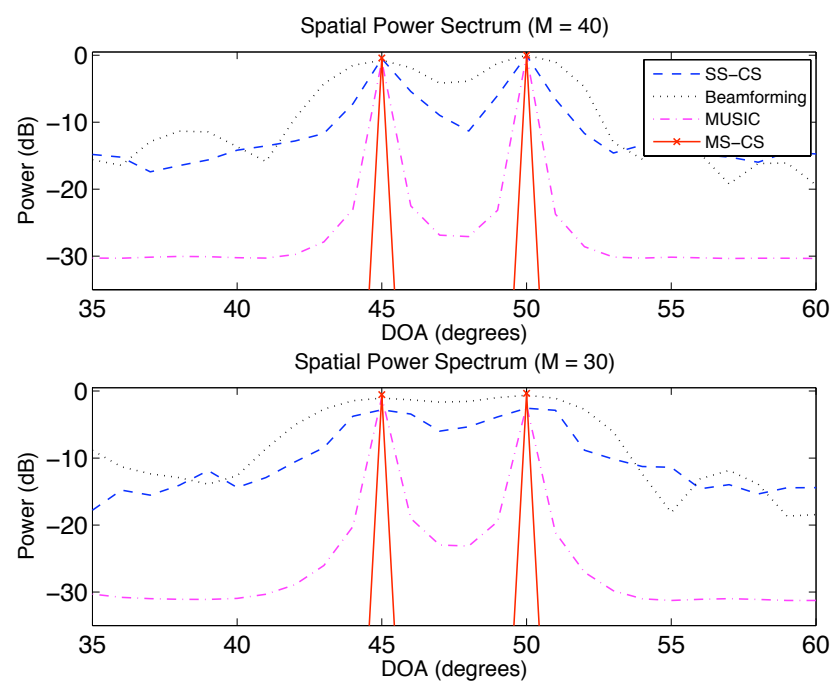

Fig. 2. Comparison of the proposed method (MS-CS) with the single channel CS (SSCS) approach, conventional beamforming and MUSIC algorithm. The number of sensors is decreased from $M=40$ to $M=30$. The SNR is $10 \mathrm{~dB}$.

\section{Conclusions}

We have proposed a multi-channel spatial compressed sensing technique that approaches the problem of source localization as a multiple vector sparse recovery problem with common sparse support. The proposed method is based on the fact that the unknown DOAs share the same sparse support, as plane waves impinge on the array from fixed locations. Simultaneous Orthogonal Matching Pursuit is used to solve the mixed norm minimization problem and assumes that the standard deviation of the additive noise is known. We have shown that the exploitation of this joint sparsity results in high resolution performance. Simulations show that our MS-CS method outperforms the standard compressed sensing method and other spectral based approaches such as beamforming or MUSIC algorithm.

Interestingly, although the worst case theoretical analysis of compressed sensing results in equivalence between the single and multi-channel case, simulations show that joint sparsity provides performance gain. This is in agreement with the average case analysis, which typically happens to be the case in the problem of sparse directional of arrival estimation, and provides the theoretical explanation on the decrease of performance of the single channel method when the number of sensors decreased.

Our future work will investigate the applicability of the proposed method in the case of a room acoustics environment and focus on the development of the MS-CS version in the near-field scenario for wideband sources. 


\section{References}

1. H. Krim and M. Viberg, Two decades of array signal processing research: the parametric approach, Signal Processing Magazine, IEEE, vol. 13, no. 4, pp. 67 94, jul 1996.

2. D. Malioutov, M. Cetin, and A.S. Willsky, A sparse signal reconstruction perspective for source localization with sensor arrays, Signal Processing, IEEE Transactions on, vol. 53, no. 8, pp. 3010 3022, aug. 2005.

3. I. Bilik, Spatial compressive sensing approach for eld directionality estimation., in Radar Conference, 2009 IEEE, may 2009, pp. 15.

4. M.F. Duarte, S. Sarvotham, D. Baron, M.B. Wakin, and R.G. Baraniuk, Dis- tributed compressed sensing of jointly sparse signals, in Signals, Systems and Computers, 2005. Conference Record of the Thirty-Ninth Asilomar Conference on, 28 november 1, 2005, pp. 15371541.

5. J.A. Tropp, A.C. Gilbert, and M.J. Strauss, Simultaneous sparse approximation via greedy pursuit, in Acoustics, Speech, and Signal Processing, 2005. Proceedings. (ICASSP 05). IEEE International Conference on, march 2005, vol. 5, pp. v/721 v/724 Vol. 5

6. S.G. Mallat and Zhifeng Zhang, Matching pursuits with time-frequency dictio- naries, Signal Processing, IEEE Transactions on, vol. 41, no. 12, pp. 3397 3415, dec 1993.

7. Y.C. Eldar and H. Rauhut, Average case analysis of multichannel sparse recovery using convex relaxation, Information Theory, IEEE Transactions on, vol. 56, no. 1, pp. 505 519, jan. 2010 .

8. E.J. Candes and M.B. Wakin, An introduction to compressive sampling, Signal Processing Magazine, IEEE, vol. 25, no. 2, pp. 21 30, march 2008.

9. E.J. Candes and T. Tao, Near-optimal signal recovery from random pro jections: Universal encoding strategies?, Information Theory, IEEE Transactions on, vol. 52, no. 12 , pp. 54065425 , dec. 2006.

10. R. Gribonval, H. Rauhut, K. Schnass, and P. Vandergheynst, Atoms of all channels, unite! Average case analysis of multi-channel sparse recovery using greedy algorithms, Journal of Fourier analysis and Applications, vol. 14, no. 5, pp. 655 687, 2008.

11. S.S. Chen, D.L. Donoho, and M.A. Saunders, Atomic decomposition by basis pursuit, SIAM review, vol. 43, no. 1, pp. 129159, 2001.

12. M. Elad and M. Aharon, Image denoising via learned dictionaries and sparse representation, in Computer Vision and Pattern Recognition, 2006 IEEE Computer Society Conference on, june 2006, vol. 1, pp. 895900. 